\title{
Predictors of Failed Intrauterine Balloon Tamponade in the Management of Severe Postpartum Hemorrhage
}

\begin{abstract}
Congcong Liu ${ }^{1}$, Jinsong Gao ${ }^{* *}$, Juntao Liu ${ }^{1 *}$, Xietong Wang ${ }^{2}$, Jing He ${ }^{3}$, Jingxia Sun ${ }^{4}$, Xiaowei Liu $^{5}$ and Shixiu Liao ${ }^{6}$

${ }^{1}$ Department of Obstetrics and Gynecology, Peking Union Medical College Hospital, Peking Union Medical College, Chinese Academy of Medical Sciences, Beijing, China, ${ }^{2}$ Department of Obstetrics and Gynecology, Shandong Provincial Hospital Affiliated to Shandong University, Jinan, China, ${ }^{3}$ Department of Obstetrics and Gynecology, Women's Hospital, School of Medicine, Zhejiang University, Hangzhou, China, ${ }^{4}$ Department of Obstetrics and Gynecology, The First Clinical Hospital Affiliated to Harbin Medical University, Harbin, China, ${ }^{5}$ Department of Obstetrics and Gynecology, Beijing Obstetrics and Gynecology Hospital, Capital Medical University, Beijing, China, ${ }^{6}$ Department of Obstetrics and Gynecology, Henan Provincial People's Hospital, Zhengzhou, China
\end{abstract}

OPEN ACCESS

Edited by:

Stefano Cianci,

University of Messina, Italy

Reviewed by:

Aida Kalok

National University of

Malaysia, Malaysia

Shuhaila Ahmad,

National University of

Malaysia, Malaysia

*Correspondence:

Jinsong Gao

gaojingsong@pumch.cn

Juntao Liu

liujtpumch@sina.com

Specialty section:

This article was submitted to

Obstetrics and Gynecology,

a section of the journal

Frontiers in Medicine

Received: 20 January 2021

Accepted: 22 June 2021

Published: 15 July 2021

Citation:

Liu C, Gao J, Liu J, Wang X, He J,

Sun J, Liu X and Liao S (2021)

Predictors of Failed Intrauterine

Balloon Tamponade in the

Management of Severe Postpartum

Hemorrhage. Front. Med. 8:656422.

doi: 10.3389/fmed.2021.656422
To identify the factors predicting intrauterine balloon tamponade (IUBT) failure for severe postpartum hemorrhage (PPH) after delivery, we conducted a retrospective cohort study of women who underwent IUBT for severe PPH after delivery from October 1, 2016 until September 30, 2017. The failure of IUBT was defined as the need of additional surgical procedures or uterine embolization. A total of 99,650 deliveries occurred during the study period. Among the patients, 106 cases of severe PPH were managed with IUBT, and the global success rate was $70.8 \%$ (75/106). Least absolute shrinkage and selection operator (LASSO) regression was performed to select the potential risk factors predicting IUBT failure. The associated risk factors - obesity, multiple gestation, cesarean delivery, estimated blood loss (EBL), and placenta accreta spectrum (PAS)-were included in multivariate logistic models. Ultimately, these models identified multiple gestation, EBL, and PAS as independent risk factors for IUBT failure. In conclusion, IUBT is an effective method for severe PPH. The presence of factors affecting IUBT failure should be recognized early, and other modalities of management should be anticipated.

Keywords: intrauterine balloon tamponade, postpartum hemorrhage, hysterectomy, placenta accreta spectrum, multiple gestation

\section{INTRODUCTION}

Postpartum hemorrhage (PPH) is still a major cause of maternal mortality worldwide (1). The universal two-child policy in China has been associated with a higher proportion of elderly pregnant women, and the incidence of PPH has significantly increased (2). It is a great challenge to actively prevent and treat $\mathrm{PPH}$ and reduce maternal morbidity and mortality.

Severe PPH is defined as a blood loss of $\geq 1,000 \mathrm{ml}$ within $24 \mathrm{~h}$ after delivery (3). If patients with severe PPH are unresponsiveness to uterine massage and first-line uterotonic agents, further invasive procedures may be required. Conservative surgical procedures include intrauterine balloon tamponade (IUBT), uterine compression sutures, pelvic vessel ligation, and arterial 
embolization. Hysterectomy is performed when all conservative life-saving treatments have failed to achieve hemostasis, which is associated with high morbidity and loss of fertility (4-8).

IUBT has been used in China since 2012 and is recommended for the treatment of $\mathrm{PPH}$ in the guidelines $(3,9)$. In recent years, IUBT has been widely used as a second-line conservative management for severe $\mathrm{PPH}$ because of its relatively non-invasive nature, and the use of IUBT is associated with a reduction in the use of more invasive procedures and has few adverse effects on subsequent menstrual and reproductive function (1013). However, the application of IUBT is mostly empirical, and evidence for its recommended use is limited. In 2020, a meta-analysis showed that the total success rate of IUBT in the treatment of $\mathrm{PPH}$ is $85.9 \%$. Nevertheless, evidence on the efficacy and effectiveness of IUBT from randomized and nonrandomized studies has been contradictory (14).

Further study is needed on the application of IUBT to determine the most effective scheme and health delivery strategy. However, prospective randomized controlled trials of $\mathrm{PPH}$ management are very difficult to perform, in particular in patients with persistent bleeding. The purpose of this study was to review the application of IUBT in severe PPH to determine the risk factors associated with IUBT failure so as to guide the selection of patients with severe PPH.

\section{MATERIALS AND METHODS}

\section{Patients and Study Design}

This study was approved by the Peking Union Medical College Hospital Review Board (reference number: JS-1151). The need for written informed consent was waived because of the retrospective nature of the study. Our study was performed in compliance with the Declaration of Helsinki.

This was a retrospective cohort study among women who underwent intrauterine balloon tamponade (IUBT) with severe PPH after delivery from October 1, 2016 until September 30, 2017 in 14 representative hospitals in 10 provinces from four major economic zones in China (including two secondary and 12 tertiary hospitals, seven general hospitals, and seven women and children's hospitals). The data collection methods are described in detail elsewhere (15). In brief, we included all of the deliveries in the hospitals after 20 weeks of gestation to assess complications of delivery. Specially assigned reporting clinicians in each hospital were responsible for collecting and reporting information regarding pregnant women who delivered using a data-collection system. All of the data entered were reviewed and verified by a second person in order to ensure the accuracy of the data entered into the database. We chose eligible cases and exported them from the central database system. In order to protect the privacy of patients, personal identifying information was removed during analysis for all of the cases.

Abbreviations: BMI, body mass index; CI, confidence interval; EBL, estimated blood loss; IUBT, intrauterine balloon tamponade; LASSO, least absolute shrinkage and selection operator; OR, odds ratio; PAS, placenta accreta spectrum; PPH, postpartum hemorrhage.
All of the maternity units in the study followed the 2014 guidelines of the Chinese Medical Association of Obstetrics and Gynecology for the prevention and management of PPH. After vaginal delivery or cesarean section, patients with $\mathrm{PPH}$ were first managed by uterine massage and first-line uterotonic agents were used (oxytocin, carbetocin, prostaglandin F2 $\alpha$ Hemabate, ergometrine, and misoprostol); if these failed, IUBT was applied according to the clinical setting as decided by the attending senior obstetrician. The balloon tamponade insertion was performed by the attending senior obstetrician followed the manufacturer's recommended instructions. Its intrauterine position was monitored by abdominal ultrasound scanning. The types of IUBT used for $\mathrm{PPH}$ were not described in this study, but Bakri balloon (Cook woman's health, USA) was the most commonly used. After the operation, the color, quantity of uterine cavity drainage fluid, height of uterine fundus, and vital signs were closely monitored. Hemoglobin and coagulation function were dynamically monitored. If hemostasis was achieved, the balloon was kept inflated up to $24-48 \mathrm{~h}$. Antibiotics were routinely used after operation to prevent infections. Patients who were given IUBT when the total blood loss was $<1,000 \mathrm{ml}$ or had other invasive procedures before IUBT were excluded from this study.

Demographic and baseline clinical data were obtained from the electronic medical records system and included maternal age, gravidity, parity, multiple gestation, pregestational body mass index (BMI), assisted reproductive technique, gynecologic and obstetric history, major medical history, and major family history. The perinatal outcomes included gestational age at birth, birthweight, the method of delivery, and $\mathrm{PPH}$. The PPH outcomes included causes of PPH, estimated blood loss (EBL), transfusion of any blood products (red blood cells, platelets, fresh frozen plasma, or cryoprecipitate), invasive procedures for severe $\mathrm{PPH}$ to stop the bleeding (uterine compression sutures, pelvic artery ligation, uterine embolization, or peripartum hysterectomy), and maternal postpartum complications, such as fever, thromboembolic events, or admission to an intensive care unit.

The grading criteria of BMI were based on the recommendation of the Working Group on Obesity in China: underweight: BMI $<18.5 \mathrm{~kg} / \mathrm{m}^{2}$, normal weight: BMI 18.5-23.9 $\mathrm{kg} / \mathrm{m}^{2}$, overweight: BMI $24.0-27.9 \mathrm{~kg} / \mathrm{m}^{2}$, and obesity: BMI $\geq$ $28.0 \mathrm{~kg} / \mathrm{m}^{2}(16)$.

$\mathrm{PPH}$ is defined as a blood loss of $\geq 500 \mathrm{ml}$ within $24 \mathrm{~h}$ after vaginal delivery or $\geq 1,000 \mathrm{ml}$ after cesarean delivery. Severe $\mathrm{PPH}$ is defined as a blood loss of $\geq 1,000 \mathrm{ml}$ within $24 \mathrm{~h}$ after delivery, unresponsiveness to uterine massage and firstline uterotonic agents, and the need for additional surgical or radiological interventions including hysterectomy (3). Each patient was assigned a single primary cause of $\mathrm{PPH}$. Some patients may have more than one causes of $\mathrm{PPH}$, the main cause of bleeding was assessed by the attending senior obstetrician. Placenta abnormalities were given as the primary cause for women with placenta praevia, placenta accreta spectrum (PAS), or placental abruption. The diagnosis of PAS was made either pathologically or clinically when the placenta did not detach normally from the uterus after delivery of the fetus. "Lacerations" 
was given as the primary cause for women with lacerations of the cervix, vagina, or perineum. "Uterine atony" was given as the primary cause for women with "global" or "lower uterine segment" atony or "softness."

The primary outcome was failure of IUBT, which was defined as the need of additional invasive procedures, including conservative surgical procedures (including uterine compression sutures or pelvic vessel ligation), uterine embolization, or peripartum hysterectomy and factors of associated with IUBT failure.

\section{Statistical Analysis}

SPSS (version 25.0, Chicago, IL, USA) was used for Student's $t$-tests, chi-square tests, or Fisher's exact-probability tests. Chisquare tests or Fisher's exact-probability tests were used to compare categorical variables that were presented as counts and percentages between groups with successful and failed IUBT. Student's $t$-test was used to compare continuous variables that are presented as means \pm standard deviations. Least absolute shrinkage and selection operator (LASSO) is the most commonly used approach to perform variable selection with regularization.
LASSO regression was performed to select the potential risk factors for the prediction of IUBT failure, which were then included in multivariate logistic models. These models were constructed to estimate the risk factors of IUBT failure. Akaike information criterion (AIC) was used to evaluate the quality of the fit of the models. Area under the curve (AUC) used to assess the predictive performance of the selected model. LASSO regression was performed with the "glmnet" package in $\mathrm{R}$ (version 4.0.2). Statistical significance was accepted when the $P<0.05$.

\section{RESULTS}

A total of 99,650 deliveries occurred from October 1, 2016 to September 30, 2017. The frequency of primary PPH was $5.3 \%(n$ $=5,267)$, and the frequency of severe $\mathrm{PPH}$ was $0.9 \%(n=863)$. Among the patients, 106 cases of severe $\mathrm{PPH}$ were managed with IUBT, 20 (18.9\%) after vaginal delivery and $86(81.1 \%)$ during or after cesarean section. The global success rate was $70.8 \%(75 / 106)$ and was significantly higher after vaginal deliveries $(18 / 20,90 \%)$ than cesareans $(57 / 86,66.3 \%, P<0.05)$.

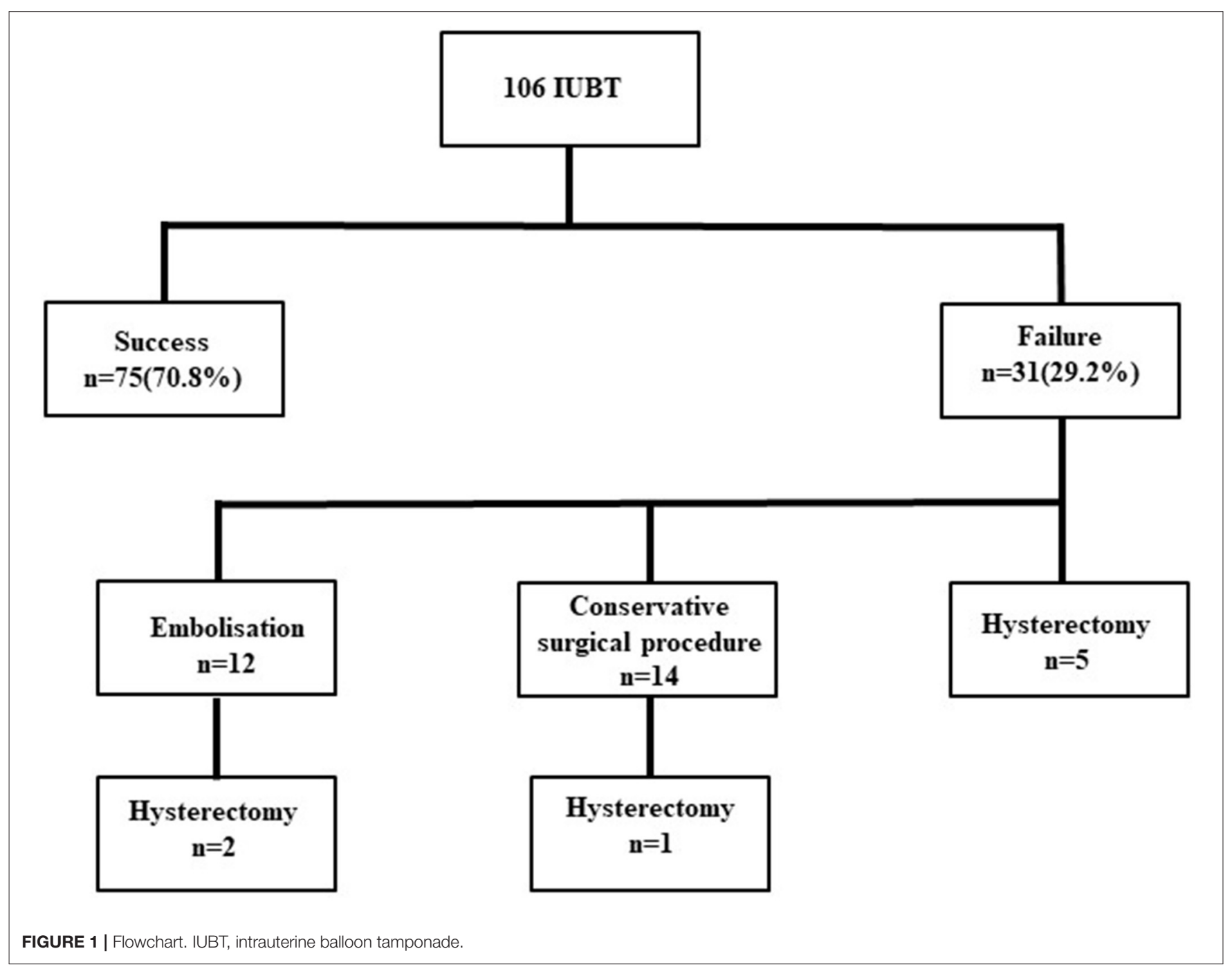


TABLE 1 | General and obstetric characteristics of the study population.

\begin{tabular}{lccc}
\hline Characteristic & Success $(\boldsymbol{n}=\mathbf{7 5})$ & Failure $(\boldsymbol{n}=\mathbf{3 1})$ & $\boldsymbol{P}$-value \\
\hline Maternal age, years* & $31.40 \pm 4.90$ & $32.29 \pm 4.13$ & 0.377 \\
$<35, n(\%)$ & $57(76.0)$ & $21(67.7)$ & 0.380 \\
$\geq 35, n(\%)$ & $18(24.0)$ & $10(32.3)$ & \\
Obesity, $n$ (\%) & $3(4.0)$ & $2(6.5)$ & 0.588 \\
Multiparous, $n$ (\%) & $33(44.0)$ & $22(71.0)$ & 0.011 \\
Parity, $n^{\star}$ & $1.64 \pm 0.77$ & $1.81 \pm 0.7$ & 0.299 \\
Preeclampsia, $n(\%)$ & $0(0.0)$ & $1(3.2)$ & 0.292 \\
Multiple gestation, $n(\%)$ & $10(13.3)$ & $5(16.1)$ & 0.707 \\
Prior cesarean delivery, $n(\%)$ & $21(28.0)$ & $19(61.3)$ & 0.001 \\
Known uterine myoma, $n(\%)$ & $2(2.7)$ & $1(3.2)$ & 1.000 \\
Assisted reproductive & $12(16.0)$ & $3(9.7)$ & 0.396 \\
technology, $n$ (\%) & & & \\
Gestational age at delivery, & $37.41 \pm 2.35$ & $36.16 \pm 2.54$ & 0.017 \\
weeks & & & \\
Mode of delivery, $n$ (\%) & & & 0.036 \\
Vaginal delivery & $18(24.0)$ & $2(6.5)$ & \\
Cesarean delivery & $57(76.0)$ & $29(93.5)$ & \\
Birthweight, $g^{*}$ & $3093 \pm 671.59$ & $2778.97 \pm 687.29$ & 0.032 \\
Birthweight $\geq 4,000 \mathrm{~g}$ & $5(6.7)$ & $1(3.2)$ & 0.486 \\
\hline
\end{tabular}

${ }^{*}$ Data are given as means \pm standard deviation.

Among the 31 failures, 12 cases underwent embolization and two cases failed, leading to hysterectomy. Fourteen other cases needed conservative surgical procedures, and one case failed, leading to hysterectomy. Hysterectomy was performed immediately after IUBT failure in five cases. There were no deaths (Figure 1).

General and obstetric characteristics of the women with IUBT are presented in Table 1. In the failure group, compared with the success group, multiparous deliveries were significantly more frequent ( 71.0 vs. $44.0 \%, P<0.05$ ), and the frequency of prior cesarean delivery was significantly higher (61.3 vs. $28.0 \%, P<$ $0.05)$. The rate of cesarean delivery in the failure group was higher (93.5 vs. $76.0 \%, P<0.05$ ). Women in the failure group delivered earlier (36.16 \pm 2.54 vs. $37.41 \pm 2.35$ weeks, $P<0.05)$ and had lower birth weights $(2778.97 \pm 687.29$ vs. $3093 \pm 671.59 \mathrm{~g}, P$ $<0.05)$ in our univariate analysis. There were no differences in other general and obstetric characteristics between the success and failure groups.

Table 2 shows the characteristics of severe PPH. The cause of severe PPH in the failure group was mainly due to PAS (17/31, $54.8 \%)$ and uterine atony $(8 / 31,25.8 \%)$ in this cohort study. There was a higher frequency of PAS ( 54.8 vs. $6.7 \%, P<0.05$ ) in the failure group. In contrast, patients with uterine atony were of lower frequency in the failure group (25.8 vs. $66.7 \%, P<$ $0.05)$. There were no significant differences in the proportions of patients with placenta previa and placental abruption between the groups. EBL was significantly higher in the failure group $(2746.13 \pm 1573.1$ vs. $1560.56 \pm 688.55 \mathrm{ml}, P<0.05)$.

The frequency of coagulopathy was significantly higher in the failure group (9.7 vs. 1.3\%, $P<0.05$ ). All coagulopathy developed due to the progression of $\mathrm{PPH}$. The volumes of packed red blood
TABLE 2 | PPH characteristics.

\begin{tabular}{lccc}
\hline Characteristic & Success $(\boldsymbol{n}=\mathbf{7 5})$ & Failure $(\boldsymbol{n}=\mathbf{3 1})$ & $\boldsymbol{P}$-value \\
\hline $\begin{array}{l}\text { Cause of hemorrhage, } n(\%) \\
\text { Uterine atony }\end{array}$ & $50(66.7)$ & $8(25.8)$ & 0.000 \\
$\begin{array}{l}\text { Placenta abnormalities } \\
\text { Placenta praevia }\end{array}$ & $18(24.0)$ & $6(19.4)$ & 0.603 \\
Placenta accreta spectrum & $5(6.7)$ & $17(54.8)$ & 0.000 \\
Placental abruption & $2(2.7)$ & $0(0.0)$ & 1.000 \\
Estimated blood loss, ml* & $1560.56 \pm 688.55$ & $2746.13 \pm 1573.1$ & 0.000 \\
\hline
\end{tabular}

PPH, postpartum hemorrhage.

${ }^{\star}$ Data are given as means \pm standard deviation.

TABLE 3 | Management of complications of PPH undergoing intrauterine balloon tamponade.

\begin{tabular}{|c|c|c|c|}
\hline Characteristic & Success $(n=75)$ & Failure $(n=31)$ & $P$-value \\
\hline $\begin{array}{l}\text { Units of red blood cells } \\
\text { transfused }\end{array}$ & $3.53 \pm 3.51$ & $7.61 \pm 5.01$ & 0.000 \\
\hline $\begin{array}{l}\text { Fresh frozen plasma } \\
\text { transfusion, } \mathrm{ml}^{\star}\end{array}$ & $289.6 \pm 316.72$ & $824.84 \pm 888.15$ & 0.003 \\
\hline Platelets transfusion, $\mathrm{U}^{\star}$ & $0.01 \pm 0.06$ & $0.1 \pm 0.4$ & 0.217 \\
\hline Coagulopathy & $1(1.3)$ & $3(9.7)$ & 0.040 \\
\hline $\begin{array}{l}\text { Admission to intensive care unit, } \\
n(\%)\end{array}$ & $11(14.7)$ & $12(38.7)$ & 0.006 \\
\hline Fever, $n(\%)$ & $1(1.3)$ & $2(6.5)$ & 0.204 \\
\hline Thromboembolic event, $n$ (\%) & $1(1.3)$ & $1(3.2)$ & 0.501 \\
\hline Maternal death, $n(\%)$ & $0(0.0)$ & $0(0.0)$ & - \\
\hline
\end{tabular}

$\mathrm{PPH}$, postpartum hemorrhage.

*Data are given as means \pm standard deviation.

cells and fresh frozen plasma transfusions were significantly higher in the failure group. Patients who underwent intensive care unit admission were more common in the failure group (38.7 vs. $14.7 \%, P<0.05$ ). No women died in the study (Table 3 ).

The list of the selected risk factors predicting IUBT failure in LASSO regression is given in Tables 1, 2. The results showed that obesity, multiple gestation, cesarean delivery, EBL, and PAS had predictive significance for IUBT failure (Figure 2). The associated risk factors were included in the logistic model. Ultimately, these models identified multiple gestation [odds ratio (OR) 15.52; 95\% confidence interval (CI) 2.27-150.454], EBL (OR 1.166; 95\% CI 1.087-1.274), and PAS (OR 26.993; 95\% CI 5.815-205.528) as independent risk factors for IUBT failure (Table 4). The AUC is 0.89 .

\section{DISCUSSION}

\section{Main Findings}

This retrospective cohort study found that the global success rate of IUBT in the management of severe PPH was $70.8 \%(75 / 106)$. We demonstrated that multiple gestation, EBL, and PAS were risk factors for IUBT failure. 
A

$\begin{array}{lllllllllllllllllllllllllllllll}22 & 22 & 22 & 22 & 21 & 20 & 19 & 18 & 18 & 19 & 19 & 19 & 19 & 18 & 17 & 14 & 13 & 11 & 10 & 9 & 5 & 3 & 3 & 3 & 2 & 2 & 2 & 2 & 2 & 2 & 0\end{array}$

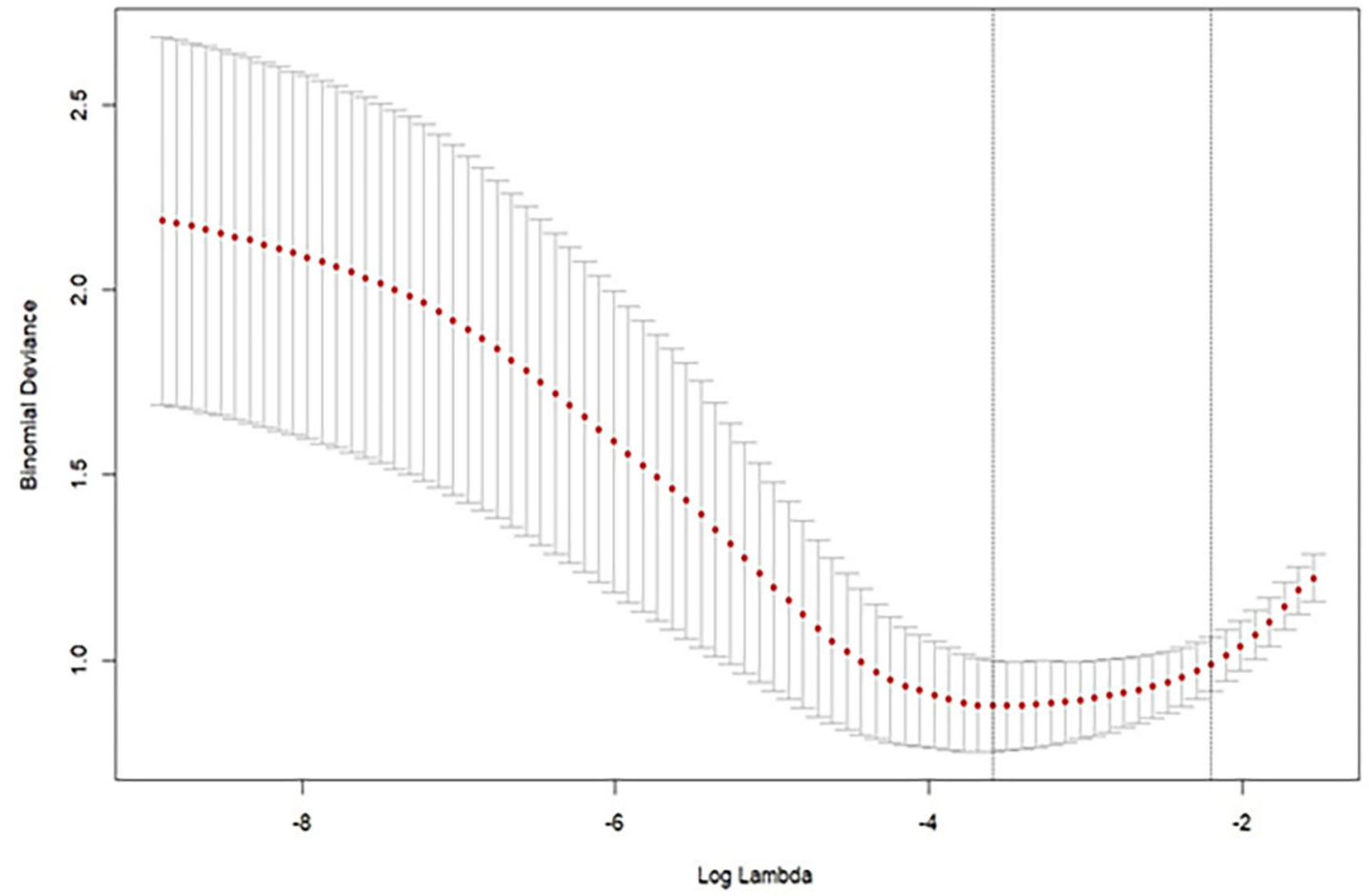

B

22

19

11

2

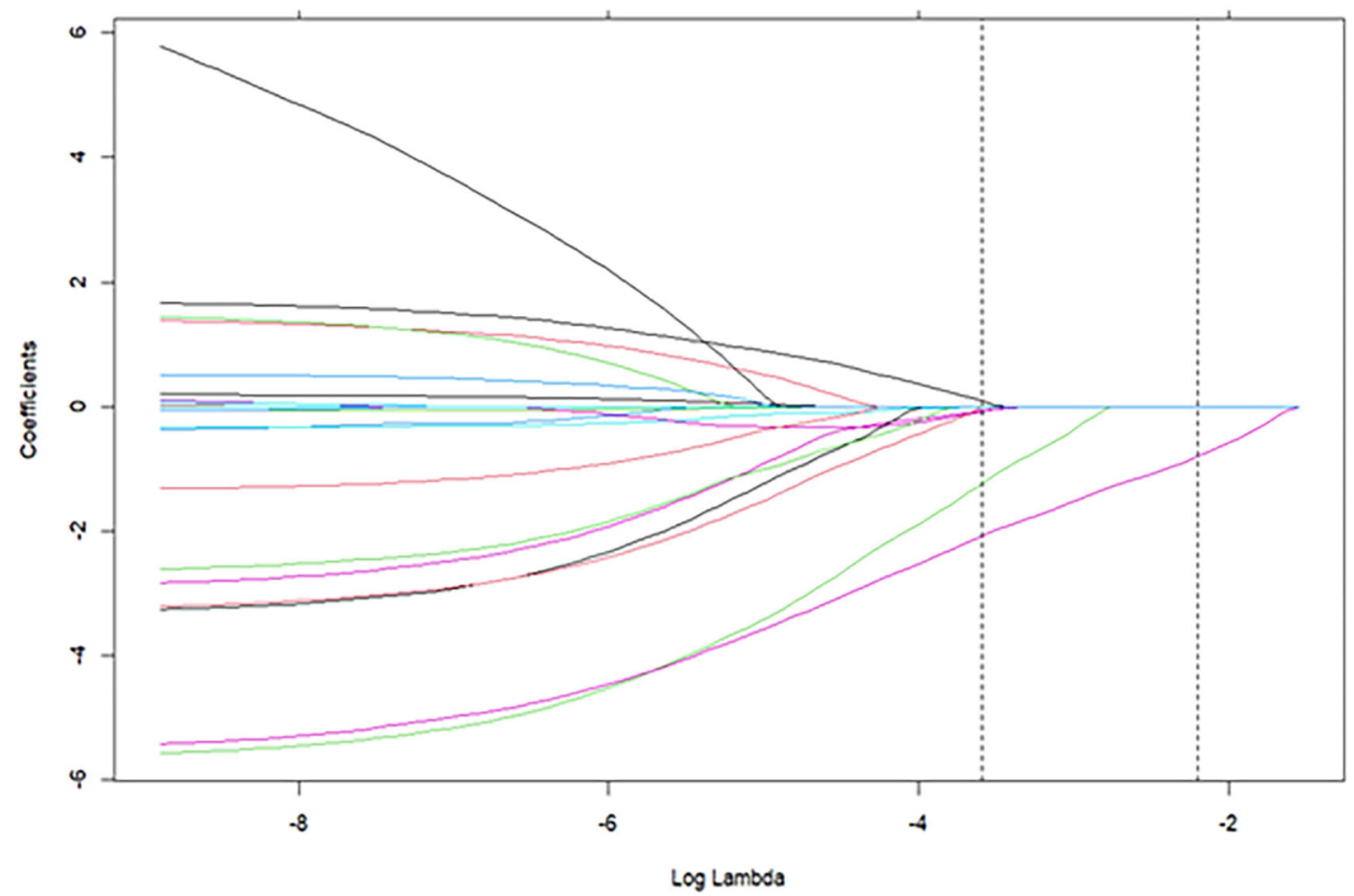

FIGURE 2 | (A) Binomial Deviance of the LASSO model with different log (lambda). (B) LASSO coefficient profiles of the risk factors predicting IUBT failure. The best combination of risk factors was selected by LASSO logistic regression analyses, with risk factors selected by the log (lambda) at which the minimal Binomial Deviance was achieved. LASSO, least absolute shrinkage and selection operator; IUBT, intrauterine balloon tamponade. 


\section{Interpretation}

The success rate of IUBT alone in the management of severe $\mathrm{PPH}$ was $70.8 \%$, which was slightly lower than in previous studies $(8,11,17)$. This may be related to the higher proportion of PAS and cesarean sections. Our study showed that the IUBT success rate for PAS was lowest among the causes of PPH. In addition, the IUBT success rate was lower for cesarean deliveries than for vaginal deliveries.

We found that PAS was associated with IUBT failure. Previous studies have demonstrated that placenta accreta is negatively associated with the success of IUBT $(11,18)$. Pala et al. (19) showed that in cases with less severe invasion and a smaller PAS area, the success rate of Bakri balloons was $84.2 \%$. Our study indicates that IUBT is not as effective for the management of severe $\mathrm{PPH}$ in patients with severe PAS in view of the high failure rate. There were 22 cases with diagnosis of PAS in the present study, and of the 17 patients with balloon failure, six cases who underwent cesarean hysterectomy had confirmed placenta increta and percreta. In cases of PAS disorders, the placenta was separating from the uterus to avoid leaving retained placental tissues in the uterine cavity, often resulting in massive obstetric hemorrhage and requiring hysterectomy. Surgical risks increased with the depth of placental invasion. Whether a balloon tamponade should be recommended in cases of PAS requires further investigation. It should be noted that IUBT is easy to deploy and the balloon can be inserted quickly. Even in cases with failure the balloon may provide a temporary tamponade effect and enough time to prepare for other interventions or patient transfer.

Our study showed that EBL was associated with IUBT failure. All of the cases involved severe PPH in this study. Women with persistent postpartum hemorrhage may develop coagulopathy with blood loss, dilution, and consumption of platelets and coagulation factors, requiring high rates of blood transfusions. Previous studies have shown that EBL before IUBT is associated with IUBT failure $(8,11,20)$, with an odds ratio for EBL before IUBT (>1,500 ml) of 3.2 (8). Howard and Grobman (21) reported that women receiving IUBT at higher EBL quartiles had more frequent packed red blood cell transfusions and more hysterectomies. IUBT needs to be used earlier in the management of persistent PPH. It is necessary to judge the bleeding characteristics and intervention timing as soon as possible, which influences the success rate of invasive procedures and impacts the prognosis of women with $\mathrm{PPH}$.

Multiple gestation is well-known to be highly associated with PPH (22, 23), which was also associated with IUBT failure in our study. In our study, multiple pregnancies had higher rates of cesarean deliveries (14/15, 93.3\%). Moreover, multiple gestations are at risk for uterine atony due to overdistension. It would seem reasonable to conclude that multiple gestations are at greater risk for IUBT failure. In addition, as previously mentioned, multiparous and prior cesarean delivery were also significantly increased in the failure group in our study. These outcomes reiterate the potential severity of PPH associated with these high-risk factors.

China's universal two-child policy announced in October 2015 has been associated with a rise in births in China and with changes in health-related birth characteristics. Between July
TABLE 4 | Multivariate regression analysis for the outcome of intrauterine balloon tamponade failure.

\begin{tabular}{lccc}
\hline Variable & $\boldsymbol{P}$ & OR & $\mathbf{9 5 \%} \mathbf{C l}$ \\
\hline Obesity & 0.534 & 1.063 & $0.880-1.301$ \\
Multiple gestation & 0.009 & 15.52 & $2.27-150.454$ \\
Estimated blood loss & $<0.001$ & 1.166 & $1.087-1.274$ \\
Cesarean delivery & 0.710 & 1.438 & $0.236-12.808$ \\
Placenta accreta spectrum & $<0.001$ & 26.993 & $5.815-205.528$ \\
\hline
\end{tabular}

OR, odds ratio; $\mathrm{Cl}$, confidence interval.

2016 and December 2017, women giving birth have been more likely to be multiparous, aged 35 and over, and the number of cesarean deliveries has risen for women who have had prior cesarean sections (2). Repeat cesarean deliveries further lead to a corresponding increase in the incidence of placenta previa and PAS (24). In 2018, national maternal monitoring found that the causes of PPH due to placental factors account for $60.49 \%$ of cases. Thus, we should increase the early identification of women at high risk of $\mathrm{PPH}$. Pregnancies at high risk for $\mathrm{PPH}$ should be transferred to a referral center for complicated pregnancies as soon as possible, and other modalities of management should be anticipated early so as to provide higher quality perinatal health care and reduce severe maternal morbidity and mortality.

In our study, the success rates of IUBT caused by uterine atony and previa were, respectively, 86.2 and $75 \%$, which is consistent with previous studies $(17,25,26)$. Therefore, we think that IUBT should be attempted for these two causes of PPH.

\section{Strengths and Limitations}

Our study was a multicenter retrospective cohort study to assess the success rate of IUBT in treating severe PPH, and all of the units that managed PPH followed the 2014 guidelines of the Chinese Medical Association of Obstetrics and Gynecology. The main limitation of the present study is its retrospective nature, which may raise some concerns regarding data quality and the risk of selection and information bias. Second, some patients may have more than one cause of $\mathrm{PPH}$, and uterine atony may be secondary to placental abnormalities and overlap each other, so it is difficult to identify the primary cause of PPH. Third, the EBL before IUBT and the interval time between the diagnosis of PPH and balloon insertion were not recorded, so we could not further evaluate when to use the IUBT. Last, the diagnosis of $\mathrm{PPH}$ can be very subjective because it is impossible to accurately measure the amount of bleeding. This will always be a limitation in $\mathrm{PPH}$ research.

\section{CONCLUSION}

In conclusion, we demonstrated that IUBT is effective when used before any invasive procedures in severe PPH. Our data demonstrated factors affecting the failure of IUBT, including multiple gestation, EBL, and PAS. The presence of factors affecting IUBT failure should be recognized early, and other modalities of management should be anticipated early on. 


\section{DATA AVAILABILITY STATEMENT}

The raw data supporting the conclusions of this article will be made available by the authors, without undue reservation.

\section{AUTHOR CONTRIBUTIONS}

JG, JL, and CL designed this study and analyzed and interpreted the data. JG, JL, XW, JH, JS, XL, and SL provided study materials or patients. CL collected data and wrote the manuscript. All authors discussed the results, contributed to the article, and approved the final manuscript.

\section{REFERENCES}

1. Say L, Chou D, Gemmill A, Tunçalp Ö, Moller A-B, Daniels J, et al. Global causes of maternal death: a WHO systematic analysis. Lancet Glob Health. (2014) 2:e323-33. doi: 10.1016/s2214-109x(14)70227-X

2. Li H-T, Xue M, Hellerstein S, Cai Y, Gao Y, Zhang Y, et al. Association of China's universal two child policy with changes in births and birth related health factors: national, descriptive comparative study. BMJ. (2019) 366:14680. doi: 10.1136/bmj.14680

3. Obstetrics Subgroup-Chinese Society of Obstetrics and GynecologyChinese Medical Association. Guideline of prevention and treatment about postpartum hemorrhage 2014. Chin J Obstet Gynecol. (2014) 49:641-6. doi: 10.3760/cma.j.issn.0529-567x.2014.09.001

4. B-Lynch C, Coker A, Lawal AH, Abu J, Cowen MJ. The B-Lynch surgical technique for the control of massive postpartum haemorrhage: an alternative to hysterectomy? Five cases reported. BJOG. (1997) 104:3725. doi: 10.1111/j.1471-0528.1997.tb11471.x

5. Joshi VM, Otiv SR, Majumder R, Nikam YA, Shrivastava M. Internal iliac artery ligation for arresting postpartum haemorrhage. BJOG. (2007) 114:35661. doi: 10.1111/j.1471-0528.2006.01235.x

6. Zwart JJ, Dijk PD, van Roosmalen J. Peripartum hysterectomy and arterial embolization for major obstetric hemorrhage: a 2-year nationwide cohort study in the Netherlands. Am J Obstet Gynecol. (2010) 202:150.E17. doi: 10.1016/j.ajog.2009.09.003

7. Kayem G, Kurinczuk JJ, Alfirevic Z, Spark P, Brocklehurst P, Knight M. Uterine compression sutures for the management of severe postpartum hemorrhage. Obstet Gynecol. (2011) 117:14-20. doi: 10.1097/aog.0b013e318202c596

8. Revert M, Cottenet J, Raynal P, Cibot E, Quantin C, Rozenberg P. Intrauterine balloon tamponade for management of severe postpartum haemorrhage in a perinatal network: a prospective cohort study. BJOG. (2017) 124:125562. doi: 10.1111/1471-0528.14382

9. Committee on Practice Bulletins-Obstetrics. Practice bulletin No. 183: postpartum hemorrhage. Obstet Gynecol. (2017) 130:e16886. doi: 10.1097/aog.0000000000002351

10. Gauchotte E, De La Torre M, Perdriolle-Galet E, Lamy C, Gauchotte G, Morel O. Impact of uterine balloon tamponade on the use of invasive procedures in severe postpartum hemorrhage. Acta Obstet Gynecol Scand. (2017) 96:87782. doi: 10.1111 /aogs. 13130

11. Kong CW, To WWK. Prognostic factors for the use of intrauterine balloon tamponade in the management of severe postpartum hemorrhage. Int $J$ Gynaecol Obstet. (2018) 142:48-53. doi: 10.1002/ijgo.12498

12. Ishii T, Sawada K, Koyama S, Isobe A, Wakabayashi A, Takiuchi T, et al. Balloon tamponade during cesarean section is useful for severe post-partum hemorrhage due to placenta previa. J Obstet Gynaecol Res. (2012) 38:1027. doi: 10.1111/j.1447-0756.2011.01625.x

13. Kong CW, To WWK. Menstrual and reproductive outcomes after use of balloon tamponade for severe postpartum hemorrhage. BMC Pregnancy Childbirth. (2018) 18:451. doi: 10.1186/s12884-018-2085-6

\section{FUNDING}

This work was supported by the 13th Five-Year National Science and Technology Support Program (No. 2015BAI13B04), the Chinese Academy of Medical Sciences (CAMS) Innovation Fund for Medical Science (CIFMS, No. 2017-I2M-3-007), and National Key R\&D Program of China (No. 2019YFC1005105).

\section{ACKNOWLEDGMENTS}

We thank all patients contributing to this study, and all the 14 representative hospitals contributing the database system.

14. Suarez S, Conde-Agudelo A, Borovac-Pinheiro A, Suarez-Rebling D, Eckardt $\mathrm{M}$, Theron $\mathrm{G}$, et al. Uterine balloon tamponade for the treatment of postpartum hemorrhage: a systematic review and meta-analysis. Am J Obstet Gynecol. (2020) 222:293.E1-52. doi: 10.1016/j.ajog.2019.11.1287

15. Zhuang C, Gao J, Liu J, Wang X, He J, Sun J, et al. Risk factors and potential protective factors of pregnancy-induced hypertension in China: a cross-sectional study. J Clin Hypertens (Greenwich). (2019) 21:61823. doi: $10.1111 /$ jch.13541

16. Zhou B-F, Cooperative Meta-Analysis Group of the Working Group on Obesity in China. Predictive values of body mass index and waist circumference for risk factors of certain related diseases in Chinese adults-study on optimal cut-off points of body mass index and waist circumference in Chinese adults. Biomed Environ Sci. (2002) 15:83-96. doi: 10.1046/j.1440-6047.11.s8.9.x

17. Mathur $\mathrm{M}, \mathrm{Ng}$ QJ, Tagore S. Use of Bakri balloon tamponade (BBT) for conservative management of postpartum haemorrhage: a tertiary referral centre case series. J Obstet Gynaecol. (2018) 38:66-70. doi: 10.1080/01443615.2017.1328671

18. Maher MA, Abdelaziz A. Comparison between two management protocols for postpartum hemorrhage during cesarean section in placenta previa: balloon protocol versus non-balloon protocol. J Obstet Gynaecol Res. (2017) 43:44755. doi: 10.1111/jog.13227

19. Pala S, Atilgan R, Başpinar M, Kavak EÇ, Yavuzkir S, Akyol A, et al. Comparison of results of Bakri balloon tamponade and caesarean hysterectomy in management of placenta accreta and increta: a retrospective study. J Obstet Gynaecol. (2018) 38:194-9. doi: 10.1080/01443615.2017.1340440

20. Grange J, Chatellier M, Chevé M-T, Paumier A, Launay-Bourillon C, Legendre G, et al. Predictors of failed intrauterine balloon tamponade for persistent postpartum hemorrhage after vaginal delivery. PLoS ONE. (2018) 13:e0206663. doi: 10.1371/journal.pone.0206663

21. Howard TF, Grobman WA. The relationship between timing of postpartum hemorrhage interventions and adverse outcomes. Am J Obstet Gynecol. (2015) 213:239.E1-3. doi: 10.1016/j.ajog.2015.04.017

22. Santana DS, Cecatti JG, Surita FG, Silveira C, Costa ML, Souza JP, et al. Twin pregnancy and severe maternal outcomes: the World Health Organization multicountry survey on maternal and newborn health. Obstet Gynecol. (2016) 127:631-41. doi: 10.1097/aog.0000000000001338

23. Madar H, Goffinet F, Seco A, Rozenberg P, Dupont C, DeneuxTharaux C. Severe acute maternal morbidity in twin compared with singleton pregnancies. Obstet Gynecol. (2019) 133:114150. doi: 10.1097/aog.0000000000003261

24. Silver RM, Landon MB, Rouse DJ, Leveno KJ, Spong CY, Thom EA, et al. Maternal morbidity associated with multiple repeat cesarean deliveries. Obstet Gynecol. (2006) 107:1226-32. doi: 10.1097/01.aog.0000219750.79480.84

25. Son M, Einerson BD, Schneider P, Fields IC, Grobman WA, Miller ES. Is there an association between indication for intrauterine balloon tamponade and balloon failure? Am J Perinatol. (2017) 34:164-8. doi: 10.1055/s-0036-1585084 
26. Cho HY, Park YW, Kim YH, Jung I, Kwon J-Y. Efficacy of intrauterine Bakri balloon tamponade in cesarean section for placenta previa patients. PLoS ONE. (2015) 10:e0134282. doi: 10.1371/journal.pone.0134282

Conflict of Interest: The authors declare that the research was conducted in the absence of any commercial or financial relationships that could be construed as a potential conflict of interest.
Copyright (C) $2021 \mathrm{Liu}, \mathrm{Gao}, \mathrm{Liu}$, Wang, He, Sun, Liu and Liao. This is an open-access article distributed under the terms of the Creative Commons Attribution License (CC $B Y)$. The use, distribution or reproduction in other forums is permitted, provided the original author(s) and the copyright owner(s) are credited and that the original publication in this journal is cited, in accordance with accepted academic practice. No use, distribution or reproduction is permitted which does not comply with these terms. 